\title{
Erratum to: Increased glucose metabolism by FDG-PET correlates with reduced tumor angiogenesis in oral squamous cell carcinoma
}

\author{
Mikiko Nakamura • Yoshimasa Kitagawa • Yutaka Yamazaki - Hironobu Hata • \\ Motoko Kotsuji • Yasuhisa Fujibayashi · Hidehiko Okazawa • Yoshiharu Yonekura • \\ Kazuo Sano
}

Published online: 31 July 2011

(C) The Society of The Nippon Dental University 2011

\section{Erratum to: Odontology}

DOI 10.1007/s10266-011-0024-3

The article cited above was incorrectly categorized. The correct category is Original Article, not Review Article.

The online version of the original article can be found under doi:10.1007/s10266-011-0024-3.

M. Nakamura $\cdot$ M. Kotsuji · K. Sano

Division of Dentistry and Oral Surgery,

Department of Sensory and Locomotor Medicine,

School of Medicine, University of Fukui, Fukui, Japan

Y. Kitagawa $(\bowtie) \cdot$ Y. Yamazaki $\cdot$ H. Hata

Oral Diagnosis and Medicine, Department of Oral

Pathobiological Science, Graduate School of Dental Medicine,

Hokkaido University, North 13, West 7, Kita-ku,

Sapporo 060-8586, Japan

e-mail: ykitagaw@den.hokudai.ac.jp

Y. Fujibayashi · H. Okazawa

Biomedical Imaging Research Center, University of Fukui,

Fukui, Japan

Y. Fujibayashi · Y. Yonekura

National Institute of Radiological Sciences, Chiba, Japan 